\title{
Réglage de la fréquence dans les réseaux électriques interconnectés
} \section{Frequency control in interconnected electricity networks}

\author{
PAR R. MEYER \\ ING. AUX B́TABLISSEMENTS NEVRUL:. \\ ProF, DE GËGULATION A L'E.N.S.H.E.G.
}

\begin{abstract}
Des questions telles que « Le couplage de deux centrales ou réseanx partiels réglant isolément la fréquence de façon stable est-il stable? 》 ou * Quels sont les centrales a mettre sur limitenr d'ouverture pour obtenir un bon réglage de la fréquence dans un réseau interconnecté?» on «Une centrale nouvelle projetée dans un réseau rendra-t-elle le réglage meilleur ou pire? "n'ont pas eu de ríponse à l'heure actuelle (sauf dans de rares cas particuliers donnés numériquement). Le but de cet article est justement de fournir lat réponse générale da de telles questions.

Dans la présente promière partie nous présenterons la méthode d'etude que nous emploierons et nous en donnerons quelques applicafions élémentaires, mais déjà très intéressantes. Par la suite nous généraliserons la méthode en ćliminant les hypotheses simplificatrices qui ont servi à alléger la présentation de la mélhod? d'étude et nous étudierons d'une façon systématique le réglage de la fréquence dans les résemux interconnectés.
\end{abstract}

\begin{abstract}
"When two power stations or partial networks having independant stable frequencly control are connected together, is the resulting system stable?" or "Which power stations have to be controlled by the gate-limit devices fo ensure a satisfactorl frequencl control in interconnected networks?" or "Will the addition of a neto power station make the frequency control more accurate or less accurate?" are questions which up to now have not been answered (except in a few special cases treated numerically). This article is intended to give a general answer to such questions.

In this the first instalment, we indicate the method of investigation used and apply it to a few elementary cases which ure nevertheless of great interest. Next we generalize the methorl by eliminating hypotheses which were used to simplify its description and then we make a systematic study of frequency control in interconnected networks.
\end{abstract}

\section{SOMMAIRE DE LA PREMIERE PARTIE}

I. -.- INTRODUCTION.

II. - DESCRIPTION DE La MÉTHODE EMPLOVÉE.

III. - Description de: L'application de la méthode aux réseaux électrigues INTERCONNECTÉS.

IV. - Applications pratioues: a) Exemple numérique; b) Couplage de deux générateurs d'un type donné; c) Couplage de nombreux générateurs du type précédent; $d$ ) Qualités du réglage dans les réseaux du type étudié précédemment; e) Méthode de la courbe symétrique.

V. - Conclusions de la première partie.

\section{PREMIERE PARTIE}

\section{I. - INTRODUCTION}

M. François Cahtex, Directeur Adjoint des Etudes et Recherches de I'Electricité de France, a écrit dans un récent rapport au Congrès de Londres de l'Union Internationale des Producteurs et Distributenrs d'Energie Electrique:

«Sur le plan technique, ce problème, (il s'agit de celui « du réglage de la frécuence et des échanges de puissance $\gg$ ) est le plus important parmi ceux que peut poser la marche en parallèle des réseaux de deux on plusieurs pays; la fréquence est en effet la même partout, de sorte qu'une coordination des réglages exécutés dans les différents réscaux est une nécessité absolue; par ailleurs, on doit pouvoir régler les puissances échangées entre ces réseaux suivant des programmes établis d'un commun accord entre les intéressés. »

Néanmoins, les méthodes de réglage de la fréquence des réseaux interconnectés et des puissances d'échange entre ces réseaux ne se basent jusqu'à présent que sur peu de connaissances théoriques et (à tort ou à raison) sur des notions mises en évidence par la thćorie du réglage de la fréquence des réseaux ne contenant qu'un seul générateur d'électricité (couramment appe- 
lés : réseaux séparés ou isolés). Cette théorie a en effet attcint à l'heure actuelle un degré de perfection très élevé, alor's qu'il n'existe aucune théorie analogue concernant les réseaux interconnectés.

Le but du présent article est d'exposer une théorie du réglage de la fréquence dans ces réseaux interconnectés.
La première partie de cet article (publié ciaprès) est destinée à décrire les principes de base de la méthode et à montrer un certain nombre d'exemples d'applications. Les autres parties comprennent des développements ultérieurs et aussi l'ensemble des justifications théoriques.

\section{1. - DESCRIPTION DE LA MÉTHODE EMPLOYEE}

Pour décrire cette méthode, nous considérerons un réseau électrique ne contenant qu'un seul générateur d'électricité. Ce cas ne servira évidemment que d'exemple; il ne présente pratiquement plus aucune difficulté théorique à l'heure actuelle.

Pour commencer, nous considérerons plus particulièrement la centrale génératrice schématisée sur la figure 1 et ferons les hypothèses suivantes, simplifiant les calculs de ce paragraphe de présentation :

Le nombre $a \mathrm{~V}_{m} / 2 g \mathrm{H}_{0}$ est nettement supérieur à 1 pour que la théorie du coup de bélier en masse soit applicable.

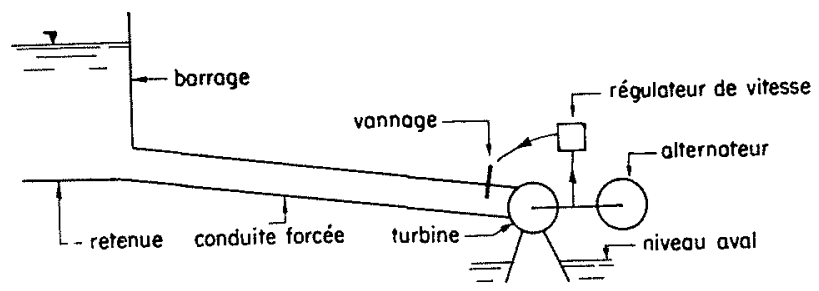

Fig. 1

La turbine est du type Francis ayant un « $n_{s}$ 》 de l'ordre de 250 à 300 , pour que son débit soit indépendant de $\Omega$ pour une position donnée du vannage.

$\Omega$ : vitesse de rotation du groupe $=(2 \pi / 60) n$ ( $n$ : nombre de tours/minute);

$H$ :charge sous laquelle débite la turbine; $n_{s}=\frac{n W^{1 / 2}}{H^{n / 4}}: \begin{gathered}\text { nombre de tours spécifique d'une tur- } \\ \text { bine; }\end{gathered}$

$\mathrm{W}$ :puissance utilisable de la turbine;

$a$ : cólérité des ondes de coup de bélier (de l'ordre de $1.000 \mathrm{~m} / \mathrm{s}$ );

$\mathrm{V}_{m}$ : itesse moyenne de l'eau dans la conduite forcée pour le débit maximum (de l'ordre de 3 à $5 \mathrm{~m} / \mathrm{s}$ );

$g=, 81 \mathrm{~m} / \mathrm{s}^{2}$ : accélération de la pesanteur;

$\mathrm{H}_{0}$ : :hute nette (environ $95 \%$ de la chute brute).

La théorie du coup de bélier en masse est en moyenne applicable pour $\mathrm{H}_{0}<100 \mathrm{~m}$.
Les turbines répondant à la condition ci-dessus sont en général employées pour des chutes de l'ordre de $40 \mathrm{~m}$ à $80 \mathrm{~m}$.

Les équations régissant les phénomènes de rém glage de la vitesse sont alors :

a) Variation de L'Énergie cinlétique du rotor (accélération du rotor) :

$$
\frac{d}{d t}\left(1 / 2 \mathrm{I} \Omega^{2}\right)=\mathrm{W}_{m}-\mathrm{W}_{r}
$$

I : moment d'inertie du rotor du groupe;

$\mathrm{W}_{m}$ : puissance motrice, fournie par l'eau;

$W_{r}$ : puissance résistante, absorbée par le réseau électrique.

b) Relation Établie par UN RÉgulateur D'UN TYPE HABITELIEMENT EMPLOYÉ, mais fortement schématisé quant à son fonctionnement (cette schématisation est à la base de presque toutes les études théoriques; nous reviendrons plus tard sur ce point) :

$$
\frac{d \mathbf{Y}}{d t}=-\frac{1}{\sigma \mathrm{T}_{r}} \frac{\Omega-\Omega_{0}}{\Omega_{0}}-\frac{1}{\sigma} \frac{d}{d t}\left(\frac{\Omega-\Omega_{0}}{\Omega_{0}}\right)
$$

$\mathrm{Y}$ : ouverture relative du vannage de la turbine;

$\Omega_{0}$ : vitesse de rotation correspondant à la fréquence de consigne;

$\sigma$ : statisme temporaire du régulateur;

$\mathrm{T}_{r}$ : temps de relaxation du dash-pot du régulateur.

Dans le cas des régulateurs accélérotachymétriques (également fortement schématisés), la même relation est valable; il convient néanmoins de remplacer $1 /\left(\sigma \mathrm{T}_{r}\right)$ par $K_{0}$ et $1 / \sigma$ par $K_{1}$.

c) DÉbtTance DE LA TURBINE :

$$
\mathrm{Q}=\mathrm{Q}_{n} \mathrm{Y} \sqrt{\mathrm{H}}
$$

Q : débit instantané;

$\mathrm{Q}_{n}$ : débit maximum. 
d) Puissance délivrée par la turbine au rotor DU GROUPE :

$$
\mathrm{W}_{m}=\bar{\omega} \eta \mathrm{QH}
$$

$\bar{\omega}$ : poids spécifique de l'eau $\left(=1.000 \mathrm{~kg} / \mathrm{m}^{3}\right)$;

$\eta$ : rendement.

e) COUP DE BÉLIER :

$$
\mathrm{H}=\mathrm{H}_{0}-\frac{d \mathrm{Q}}{d t} \frac{1}{g} \sum_{i} \frac{\mathrm{L}_{i}}{s_{i}}=\mathrm{H}_{0}-\frac{\mathrm{L}}{g s} \frac{d \mathrm{Q}}{d t}
$$
$\underset{i}{\Sigma} \frac{\mathrm{L}_{i}}{s_{i}}$ : somme des longueurs du trajet de l'eau respondantes.

Le trajet de l'eau peut comprendre un ou plusieurs tronçons de conduite forcée de sections différentes, la bâche de la turbine, la turbine, l'aspirateur et, éventuellement, une conduite de fuite en charge à l'aval de la turbine.

$$
\frac{\mathrm{L}}{s}=\Sigma_{i} \frac{\mathbf{L}_{i}}{s_{i}}: \begin{aligned}
& \text { rapport longueur sur section d'une } \\
& \begin{array}{l}
\text { conduite équivalente du point de } \\
\text { vue du coup de bélier. }
\end{array}
\end{aligned}
$$

Pour simplifier les calculs relatifs à l'exemple d'introduction, nous admettrons en plus :

a) Qu'il est licite de remplacer les équations cidessus par des équations «linéaires »approchant au mieux les équations vraies pour les oscillations de faible amplitude (hypothèse faite dans la plupart des études théoriques de première approximation);

b) Que le rendement est approximativement constant durant les oscillations de réglage envisagées, ce qui est licite moyennant l'hypothèse $a$ ) et si le point de fonctionnement est au voisinage du sommet de la colline de rendement de la turbine;

c) Que la puissance $W_{r}$ ne varie pas du fait des variations de $\Omega$, ce qui est par exemple le cas si l'alternateur est muni d'un régulateur de tension très prompt et très puissant et si le réseau électrique a une impédance purement ohmique.

En outre, nous poserons :

$\frac{\mathrm{I} \Omega_{0}{ }^{2}}{\mathrm{~W}_{n}}=\tau:$ temps de lancer du rotor.

$W_{n}$ : puissance nominale du groupe.

$$
\frac{\Omega-\Omega_{0}}{\Omega_{0}}=\frac{f-f_{0}}{f_{0}}=\Delta f
$$

$f$ : fréquence « instantanée 》 du réseau électrique.

$f_{0}$ : fréquence de consigne.
Les régimes transitoires considérés étant très lents devant la fréquence d'environ $50 \mathrm{~Hz}$ du courant électrique, on peut définir à chaque instant une fréquence « instantanée ». On peut par exemple prendre : $f=2 \mathrm{k} \Omega\left(^{*}\right)$. $k$ : nombre de paires de pôles de l'alternateur. $\mathrm{Y}-\mathrm{Y}_{0}=y$.

$Y_{0}$ : valeur de $Y$ correspondant au régime permanent moyen autour duquel fluctuera le régime instantané pendant les oscillations de réglage.

$\frac{\mathrm{Q}-\mathrm{Q}_{0}}{\mathrm{Q}_{n}}=q, \quad$ avec $\mathrm{Q}_{0}=\mathrm{Q}_{n} \mathrm{Y}_{0}$.

$$
\begin{gathered}
\mathrm{W}_{m}-\mathrm{W}_{0}=\Delta \mathrm{W}_{m} ; \quad \mathrm{W}_{r}-\mathrm{W}_{0}=\Delta \mathrm{W}_{r} \\
\operatorname{avec} \mathrm{W}_{0}=\bar{\omega} \eta \mathrm{Q}_{0} \mathrm{H}_{0} .
\end{gathered}
$$

$\frac{\mathrm{H}-\mathrm{H}_{0}}{\mathrm{H}_{0}}=h ; \frac{\mathrm{LQ}_{n}}{g s \mathrm{H}_{0}}=\underset{\text { conduite. }}{\Theta: \text { temps de lancer de la }}$

On obtient alors :

$$
\begin{gathered}
\tau \frac{d \Delta f}{d t}=\frac{\Delta \mathrm{W}_{m n}-\Delta \mathrm{W}_{r}}{\mathrm{~W}_{n}} \\
\frac{d y}{d t}=-\frac{1}{\sigma \mathrm{T}_{r}} \Delta f-\frac{1}{\sigma} \frac{d \Delta f}{d t} \\
q=y+1 / 2 \mathrm{Y}_{0} h \\
\frac{\Delta \mathrm{W}_{m}}{\mathrm{~W}_{n}}=q+\mathrm{Y}_{0} h
\end{gathered}
$$

et :

$$
h=-\Theta \frac{d q}{d t}
$$

Seules les variations de $\Delta \mathrm{W}_{r} / \mathrm{W}_{n}$ et de $\Delta f$ sont intéressanles. En effet, ce sont les variations de $\Delta \mathrm{W}_{r} / \mathrm{W}_{n}$ qui entraînent celles de $\Delta f$ et le régulateur de vitesse a justement pour mission de maintenir $\Delta f$ aussi petit que possible, malgré les variations de $\Delta \mathrm{W}_{r} / \mathrm{W}_{n}$.

Nous allons donc éliminer toutes les autres variables. En fait, il n'est pas nécessaire de considérer tous les modes de variations possibles de $\Delta \mathrm{W}_{r}, \mathrm{~W}_{n}$. En effet, les équations étant linéaires, on peut déduire tous les résultats intéressants de la seule étude des oscillations sinusoïdales. Nous reviendrons d'ailleurs sur ee point ultérieurement. En attendant, nous étudierons donc les régimes d'oscillations sinusoïdales.

Rappelons que si les équations sont linéaires (et seulement dans ce cas d'ailleurs) toutes les grandeurs peuvent varier en même temps sinusoïdalement et avec la même pulsation. On peut donc poser a priori :

$\frac{\Delta W_{r}}{W_{n}}=\frac{\Delta W_{0}}{W_{n}} \cos (\omega t+\varphi) ; \quad \Delta f=\Delta f_{0} \cos (\omega t+\Psi) ;$

$\left.{ }^{\star}\right)$ Nous donnerons dans la $2^{c}$ partie une définition exacte de la fréquence instantanée. 
etc., et introduire ces valeurs dans les équations. En réalité, il est bien plus commode de calculer avec des vecteurs dans un plan complexe, selon la méthode de Fresnel (classique en particulier en électrotechnique des courants alternatifs). Rappelons que cette méthode de calcul se base sur les faits suivants :

a) Au lieu de poser, par exemple :

$$
\Delta f=\Delta f_{0} \cos (\omega t+\varphi)
$$

on écrit :

$$
\Delta f=\mathrm{R}\left[\Delta f_{0} e^{j \omega t} \cdot e^{j \varphi}\right]
$$

où $R[\ldots]$ signifie : partie réelle de ... et $e=2,71828 \ldots ; j=\sqrt{-1}$.

b) On pose $\Delta f_{0} c^{i \varphi}=F$ : nombre complexe dont la valeur absolue est l'amplitude de l'oscillation et l'argument le déphasage par rapport à $\cos \omega t$.

c) $\mathrm{De}:$

el :

$$
(d / d t) \mathrm{R}\left[F e^{j \omega t}\right]=\mathrm{R}\left[F j \omega e^{j \omega t}\right]
$$

$$
\mathrm{R}(\mathrm{A})+\mathrm{R}(\mathrm{B})=\mathrm{R}(\mathrm{A}+\mathrm{B}),
$$

on déduit qu'on peut mettre le symbole $\mathrm{R}$ « en facteur » devant chaque équation.

Ainsi :

$$
\tau \frac{d \Delta f}{d t}-\frac{\Delta W_{b n}-\Delta W_{r}}{W_{n}}=0
$$

peut s'écrire :

$$
\mathbf{R}\left(\left[\tau j \omega F-\frac{W_{m}-W_{r}}{W_{n}}\right] e^{j_{\omega t}}\right)=0
$$

où $W_{m}$ et $W_{r}$ sont les amplitudes complexes de $\Delta \mathrm{W}_{m}$ et $\Delta \mathrm{W}_{r}$.

$$
\text { d) De R }\left[(\alpha+b j) e^{j \omega t}\right]=0 \text {, on déduit : }
$$$$
a \cos \omega t-b \sin \omega t=0 \text {, }
$$

ce qui n'est vérifié, quel que soit $t$, que si :

$$
a=0 \text { et } b=0 \text { ou bien } a+b j=0 \text {. }
$$

On peut donc supprimer les symboles $R[\ldots]$ et le facteur $e^{j \omega t}$ et on obtient les relations entre les amplitudes complexes.

Dans le cas des équations précédentes, on obtient :

$$
\begin{gathered}
\tau j \omega F=\frac{W_{m}-W_{r}}{W_{u}} \\
j \omega Y=-\frac{1}{\sigma \mathrm{T}_{r}}\left(1+j \omega \mathrm{T}_{r}\right) F \\
Q=Y+1 / 2 \mathrm{Y}_{0} H \\
\frac{W_{m}}{W_{n}}=Q+\mathrm{Y}_{n} H \\
H=-\Theta j \omega Q
\end{gathered}
$$

où $F ; W_{m} ; W_{r} ; Y ; H$ et $Q$ sont respectivement les amplitudes complexes de $\Delta f ; \Delta W_{m} ; \Delta W_{r} ; y$ : $h$ et $q$.

L'élimination des grandeurs autres que $W_{r}$ et $F$ est maintenant aisée et donne :

$W_{r}=$

$-F \mathrm{~W}_{n}\left[\tau j \omega+\frac{1-\Theta \mathrm{Y}_{0} j \omega}{1+(1 / 2) \Theta \mathrm{Y}_{0} j \omega} \cdot \frac{1}{\sigma \mathrm{T}_{r}} \cdot \frac{1+j \omega \mathrm{T}_{r}}{j \omega}\right]$ $=-F G(j \omega)$

Nous poserons $G(j \omega)=\mathrm{X}(\omega)+j \mathrm{Z}(\omega)$ et nous appellerons $G(j \omega)$ fonction de réponse de l'installation. Elle permet en effet de déterminer la réponse $\Delta f(t)$ à une excitation due à une variation de la puissance demandée par le réseau électrique : $\Delta W_{\bullet}(t)$.

Nous verrons qu'on peut étudier entièrement le réglage, en particulier sa stabilité et ses autres qualités, en étudiant la fonction $G(j \omega)$. Pour l'instant, nous nous contenterons de l'étude de la stabilité.

Remarquons d'abord, que, si pour une valeur $\omega=\omega_{0}$, on a $X\left(\omega_{0}\right)=0$ et $Z\left(\omega_{0}\right)=0$, c'est-à-dire $G\left(j \omega_{0}\right)=0$, on peut avoir $F \neq 0$ même si $W_{r}=0$. Cela veut dire qu'il peut exister une oscillation entretenue de pulsation $\omega_{0}$ de la fréquence du courant du réseau électrique et ceci même si la puissance demandée par le réseau électrique reste constante. Dans ce cas, l'installation est à sa limite de stabilité.

La courbe paramétrique $\mathrm{X}=\mathrm{X}(\omega) ; \mathrm{Z}=\mathrm{Z}(\omega)$ tracée dans un plan $X ; Z$, s'appellera : courbe de réponse. Nous venons de voir que si cette courbe passe par l'origine pour une valeur $\omega_{0} \mathrm{du}$ paramètre $\omega$, l'installation est à la limite de stabilité. Il est intuitif de penser (et nous le démontrerons ultérieurement en toute rigueur) que, suivant la façon dont la courbe de réponse passe près de l'origine, l'installation sera stable ou non. Le critère exact est le suivant * :

Pour que l'installation soit stable, le rayon vecteur joignant l'origine au point courant de la courbe de réponse doit balayer un angle de $(a-b) \pi / 2$ quand la valcur du paramètre correspondant au point courant varie de $\omega=0$ à $\omega=+\infty, a$ et $b$ sont donnés par :

et :

$$
\lim _{\omega \rightarrow \infty} G^{x}(j \omega)=A(j \omega)^{a}
$$

$$
\lim _{b \rightarrow 0} G(j \omega)=\mathrm{B}(j \omega)^{b} .
$$

Dans l'exemple actuel, on a :

$$
\lim _{\omega \rightarrow \infty} G(j \omega)=\mathrm{w}_{n} \cdot \tau j_{\omega},
$$

(*) Un cas très particulier de ce critère est le critère dit « de Leonhard». 
donc : $a=1$

$$
\text { et } \lim _{\omega \rightarrow \infty} G(j \omega)=\frac{W_{n}}{\sigma T_{r}} \frac{1}{j \omega}
$$

donc $: b=-1$ et $(a-b) \frac{\pi}{2}=+\pi$.

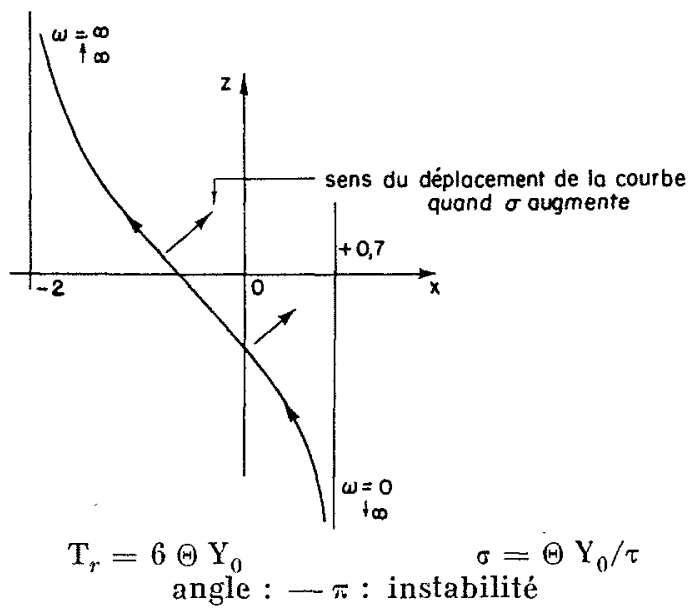

FIG. 2

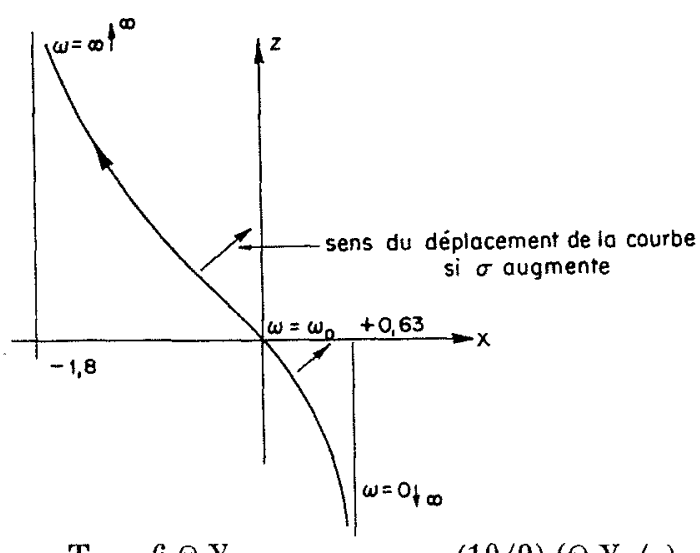

$\mathrm{T}_{r}=6 \Theta \mathrm{Y}_{0} \quad \sigma=(10 / 9)\left(\Theta \mathrm{Y}_{0} / \tau\right)$
Limite de stabilité, pulsation de l'oscillation entretenue : $\omega_{0}=\sqrt{(3 / 2)\left[1 /\left(\Theta \mathrm{Y}_{0}\right)\right]}$

FIG. 3

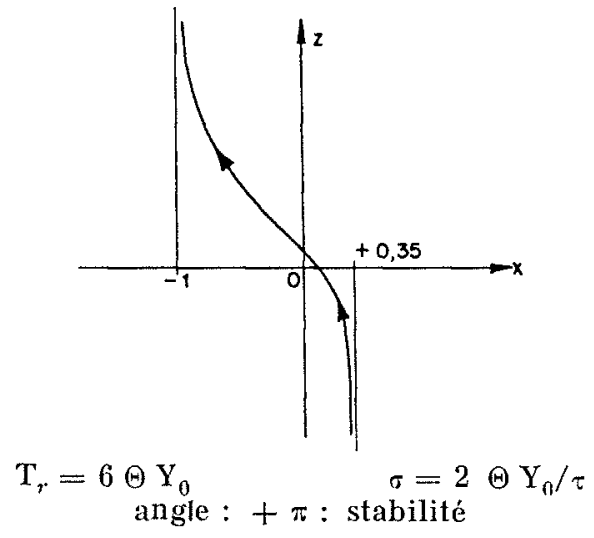

Frg. 4
Les figures 2, 3 et 4 montrent trois courbes de réponse relatives à trois installations différentes. Dans ce cas simple, on peut aussi déterminer les conditions de stabilité par la méthode d'Hurwitz, et on trouve :

$$
\mathrm{T}_{r}>0 \text { et } \sigma>\frac{\Theta \mathrm{Y}_{0}}{\tau} \frac{1-\left(\Theta \mathrm{Y}_{0} / \mathrm{T}_{r}\right)}{1-(3 / 2)\left(\Theta \mathrm{Y}_{0} / \mathrm{T}_{r}\right)}
$$

Sur ces figures, nous avons porté sur les axes les grandeurs sans dimensions $x=\left(\mathrm{X} / \mathrm{W}_{n}\right)$ et $z=\left(Z / W_{n}\right)$. En outre, nous avons muni les cour-

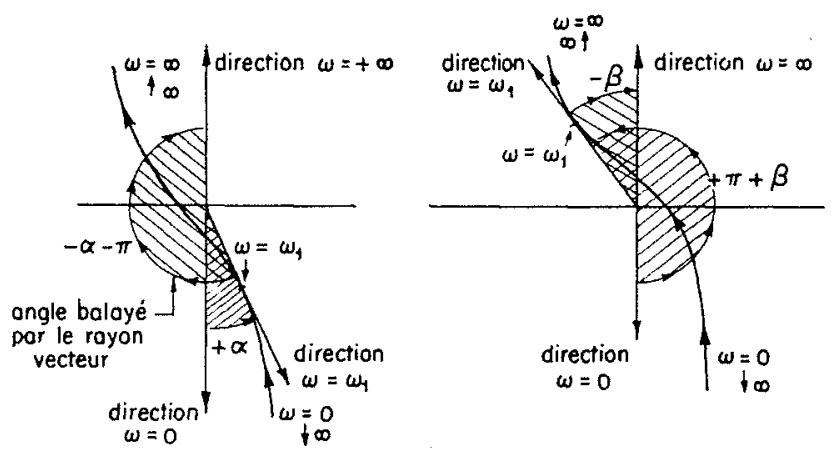

On balaye successive ment les angles :

$$
\begin{array}{cc}
+\alpha \text { et }-\alpha-\pi & +\pi+\beta \text { et }-\beta \\
\text { au total : }-\pi & \text { au total : } 1+\pi \\
\text { Cas de la figure } 2 & \text { Cas de la figure } 4
\end{array}
$$

FIG. 5

bes de réponse de flèches montrant le sens des valeurs croissantes de $\omega$ : il en sera désormais toujours ainsi. La figure 5 montre les angles balayés par le rayon vecteur. On voit de quelle facon cet angle change quand la courbe de réponse passe par l'origine lors de ses déformations dues à des variations continues des paramètres de l'installation.

Remarquons que la formule donnant $W_{r}$ en fonction de $F$ contient deux termes :

- Le premier, $W_{n} F \tau j \omega$, donne la puissance que fournit le rotor quand la vitesse de rotation du groupe varie. En effet, le rotor contient l'énergie $1 / 2$ I $\Omega^{2}$ et une variation de $\Omega$ libère la puissance $d / d t\left(1 / 2 I \Omega^{2}\right)$.

- Le deuxième :

$$
\mathrm{W}_{n} F \cdot \frac{1}{\sigma \mathrm{T}_{r}} \cdot \frac{1+j \omega \mathrm{T}_{r}}{j \omega} \cdot \frac{1-\Theta \mathrm{Y}_{0} j \omega}{1+(1 / 2) \Theta \mathrm{Y}_{0} j \omega}
$$

donne la puissance supplémentaire fournie par l'eau à l'alternateur puis au réseau par suite de l'action du régulateur de vitesse de la turbine sur le vannage quand la vitesse de rotation du groupe varie.

D’une façon générale, il peut y avoir encore un troisième terme provenant de la variation du rendement du groupe quand la vitesse de rotation varie. 


\section{III. - DESCRIPTION DE L'APPLICATION DE LA PRESENTE METHODE AUX RÉSEAUX ÉLECTRIQUES INTERCONNECTÉS}

Envisageons maintenant le réseau représenté schématiquement sur la figure 6 . Pour simplifier l'exposé, nous admettrons pour chaque générateur et récepteur les hypothèses adéquates faites au paragraphe II. En plus, nous admettrons que les impédances de liaison sont négligeables et que la tension est maintenue constante au point A.

Au paragraphe II, nous avons admis que le réseau électrique était soumis à une perturbation faisant varier la puissance demandée selon $\Delta W_{r}=\Delta W_{r 0} \cos (\omega t+\Psi)$ sans préciser la provenance de cette perturbation.

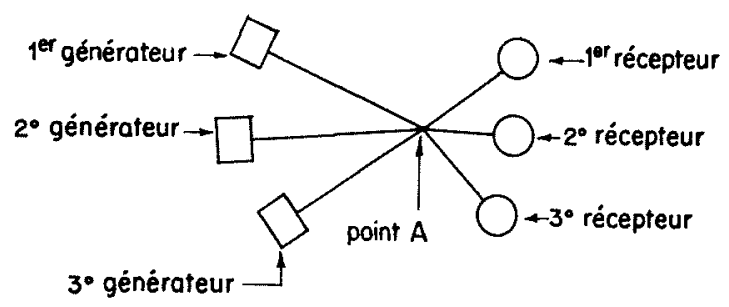

FIG. 6

Admettons ici que l'on oblige l'un des récepLeurs du réseau, par exemple le troisième, à absorber la puissance $\mathrm{W}_{30}{ }^{\prime}+\Delta \mathrm{W}_{r 0} \cos \left(\omega t+\mathrm{IP}^{\mathrm{P}}\right)$.

En vertu des hypotheses faites, on a :

$$
\mathrm{W}_{1}+\mathrm{W}_{2}+\mathrm{W}_{3}=\mathrm{W}_{1}^{\prime}+\mathrm{W}_{2}^{\prime}+\mathrm{W}_{3}^{\prime}
$$

$W_{1}: W_{2} ; W_{3}:$ puissances instantanées fournies par les générateurs.

$\mathrm{W}_{1}^{\prime} ; \mathrm{W}_{2}^{\prime} ; \mathrm{W}_{3}^{\prime}$ : puissances instanlances absorbées par les récepteurs.

Comme les équations régissant les oscillations de réglage sont supposées être linéaires, on peut à nouveau se contenter des oscillations sinusoïdales. On pourra poser :

$$
\mathrm{W}_{1}=\mathrm{W}_{10}+\Delta \mathrm{W}_{1} ; \mathrm{W}_{2}=\mathrm{W}_{20}+\Delta \mathrm{W}_{2} ; \ldots
$$

$W_{10} ; W_{20} ; \ldots$ : valeurs des puissances dans le cas du régime permanent autour duquel oscille le régime lors du réglage.

$\Delta \mathrm{W}_{1} ; \Delta \mathrm{W}_{\underline{2}} ; \ldots$ : oscillations dues au réglage.

On aura donc :

$$
\mathrm{W}_{10^{\prime}}+\mathrm{W}_{20^{\prime}}+\mathrm{W}_{30^{\prime}}=\mathrm{W}_{10}+\mathrm{W}_{20}+\mathrm{W}_{30}
$$

et :

$$
\Delta \mathrm{W}_{1}+\Delta \mathrm{W}_{2}+\Delta \mathrm{W}_{3}=\Delta \mathrm{W}_{1}^{\prime}+\Delta \mathrm{W}_{2}^{\prime}+\Delta \mathrm{W}_{3}^{\prime}
$$

Les oscillations de charge $\Delta \mathrm{W}_{3}^{\prime}$ du troisième générateur feront osciller la fréquence dans tout le réseau. L'oscillation de fréquence sera liée aux oscillations des diverses puissances produites ou absorbées. Ainsi on a vu au paragraphe II que si la fréquence oscillait, la puissance du générateur de la figure 1 oscillait selon :

$$
\begin{aligned}
& W= \\
& -F \mathrm{~W}_{n}\left[\tau j \omega+\frac{1-\Theta \mathrm{Y}_{0} j \omega}{1+(1 / 2) \Theta \mathrm{Y}_{0} j \omega} \frac{1}{\sigma \mathrm{T}_{r}} \frac{1+j \omega \mathrm{T}_{r}}{j \omega}\right] \\
& =-F G(j \omega)
\end{aligned}
$$

Cette oscillation n'est pas affectée par l'interconnection. Elle provient, comme on l'a expliqué au paragraphe II, d'une part $\left(W_{n} \tau j \omega\right)$ de la variation d'énergie cinétique $d u$ rotor : le rotor cède de la puissance au réseau si la fréquence diminue, donc la vitesse de rotation diminue, d'autre part, de l'action du régulateur de vitesse qui agit sur le vannage dès que la fréquence varie.

D’une façon générale, on aura dans le réseau :

$$
W_{1}=-F G_{1}(j \omega) ; W_{2}=-F G_{2}(j \omega) ; \ldots
$$

D'où :

$$
\begin{aligned}
W_{3}^{\prime}=-F\left[G_{1}(j \omega)+G_{2}(j \omega)+G_{3}(j \omega)\right. \\
\\
\left.\quad-G_{1}^{\prime}(j \omega)-G_{2}{ }^{\prime}(j \omega)\right]
\end{aligned}
$$

On appellera : $G_{1}(j \omega)+G_{2}(j \omega) \pm \ldots=G(j \omega)$ la fonction de réponse du réseau, comme $G_{1}(j \omega)$ est celle du premier générateur. De $W_{3}^{\prime}=-F G(j \omega)$, où $W_{3}^{\prime}$ est l'amplitude complexe de la variation de puissance et $F$ celle de la variation de fréquence correspondante, on peut lirer les mêmes résultats que dans le cas des réseaux séparés traité an paragraphe II.

La courbe de réponse $G(j \omega)$ s'obtient à partir de $G_{1}(j \omega) ; G_{2}(j \omega)$, par addition vectorielle; en effet, on a :

$$
\begin{aligned}
& X(\omega)=X_{1}(\omega)+X_{2}(\omega)+\ldots \\
& Z(\omega)=Z_{1}(\omega)+Z_{2}(\omega)+\ldots
\end{aligned}
$$




\section{IV. - APPLICATIONS PRATIQUES :}

a) Exemple Numérique : COUplage dE DEUX GÉNÉRATEURS DU TYPE FIGURE 1.

\section{Caractéristiques :}

Premier générateur : $\mathrm{W}_{n}=100 \mathrm{MW} ; \Theta=0,3 \mathrm{~s}$; $\tau=1,5 \mathrm{~s}$ (groupe ultra-léger, inertie de l'eau très faible).

Deuxième générateur : $W_{n}=20 \mathrm{MW} ; \Theta=2 \mathrm{~s}$; $\tau=10 \mathrm{~s} \quad\left(\mathrm{PD}^{2}\right.$ normal, inertie de l'eau grande).

Adniettons que $\sigma$ et $\mathbf{T}_{r}$ aient été choisis dans chaque cas de façon à obtenir le réglage optimum en réseau séparé; donc $\sigma \# 30 \%$ et ' $T_{r}=2 \mathrm{~s}$ et $12 \mathrm{~s}$, respectivement.

On obtient alors :

$$
\begin{aligned}
& \frac{1}{100} G_{1}(j \omega)=1,5 j \omega+\frac{1-0,3 j \omega}{1+0,15 j \omega} \cdot \frac{1}{0,6} \cdot \frac{1+2 j \omega}{j \omega} \\
& \frac{1}{20} G_{2}(j \omega)=10 j \omega+\frac{1-2 j \omega}{1+j \omega} \cdot \frac{1}{3,6} \cdot \frac{1+12 j \omega}{j \omega} \\
& \text { ou : } \\
& \frac{1}{100} G_{1}(j \omega)=1,5 j \omega-j \frac{1,66+1,43 \omega^{2}}{\left(1+0,225 \omega^{2}\right) \omega} \\
& \frac{1}{20} G_{2}(j \omega)=10 j \omega-j \frac{2,58-0,15 \omega^{2}}{1+0,0225 \omega^{2}} \\
& \begin{array}{c}
\left(1+\omega^{2}\right) \omega \\
+\frac{2,5-6,66 \omega^{2}}{1+\omega^{2}}
\end{array}
\end{aligned}
$$

La figure 7 montre les courbes avant couplage, la figure 8 la courbe résultante après couplage. Ces courbes sont graduées en $\omega$; seules, les valeurs correspondant aux graduations marquées sur les figures ont été calculées. On aurait pu en calculer moins pour résoudre le problème posé. On voit que le couplage est stable.

\section{b) Couplage De Deux Générateurs DU Type FIGURE 1, EN GÉNÉRAL.}

On peut écrire la fonction de réponse sous la forme suivante :

$$
\begin{aligned}
& \frac{1}{\mathrm{~W}_{n}} G(j \omega)=\tau j \omega+\frac{1-\mathrm{Y}_{0} \Theta j \omega}{1+(1 / 2) \mathrm{Y}_{0} \Theta j \omega} \frac{1}{\sigma \mathrm{T}_{r}} \frac{1+j \omega \mathrm{T}_{r}}{j \omega} \\
& =\frac{\tau}{\Theta \mathrm{Y}_{0}}\left[j \Theta \mathrm{Y}_{0} \omega+\frac{1-j \Theta \mathrm{Y}_{0} \omega}{1+(1 / 2) \Theta \mathrm{Y}_{0} j \omega} \frac{y+\mu j \Theta \mathrm{Y}_{n} \omega}{j \Theta \mathrm{Y}_{0} \omega}\right] \\
& \text { en posant } \mu=\frac{\Theta \mathrm{Y}_{0}}{\sigma \tau} \text { et } \nu=\frac{\Theta^{2} \mathrm{Y}_{0}^{2}}{\sigma \mathrm{T}_{r} \tau}
\end{aligned}
$$

Si on détermine $\sigma$ et $\mathrm{T}_{r}$ de facon telle que chaque groupe ait les mêmes qualités de réglage ou de stabilité en réscau séparé, les valeurs de $\mu$ et $y$ seront les mêmes pour tous les groupes (cf. annexe). Nous allons étudier ce cas.

On peut écrire:

$$
\begin{aligned}
& \mathrm{X}=\frac{\tau \mathrm{W}_{n}}{\Theta \mathrm{Y}_{0}} \frac{\mu-(3 / 2) \nu-(1 / 2) \mu\left(\Theta \mathbf{Y}_{0} \omega\right)^{2}}{1+(1 / 4)(\Theta \mathrm{Y} \omega)^{2}} \\
&=\frac{W_{n} \tau}{\Theta \mathbf{Y}_{0}} x\left(\Theta \mathrm{Y}_{0} \omega ; \mu ; \nu\right)
\end{aligned}
$$

$Z=$

$$
\begin{array}{r}
\frac{\tau W_{n}}{\Theta Y_{0}}\left[\Theta Y_{0} \omega-\frac{\nu-(1 / 2) \nu\left(\Theta Y_{0} \omega\right)^{2}+(3 / 2) \mu\left(\Theta Y_{0} \omega\right)^{2}}{\left[1+(1 / 4)\left(\Theta Y_{0} \omega\right)^{2}\right] \Theta Y_{0} \omega}\right] \\
=\frac{W_{n} \tau}{\Theta Y_{0}} z\left(\Theta Y_{0} \omega ; \mu ; v\right)
\end{array}
$$

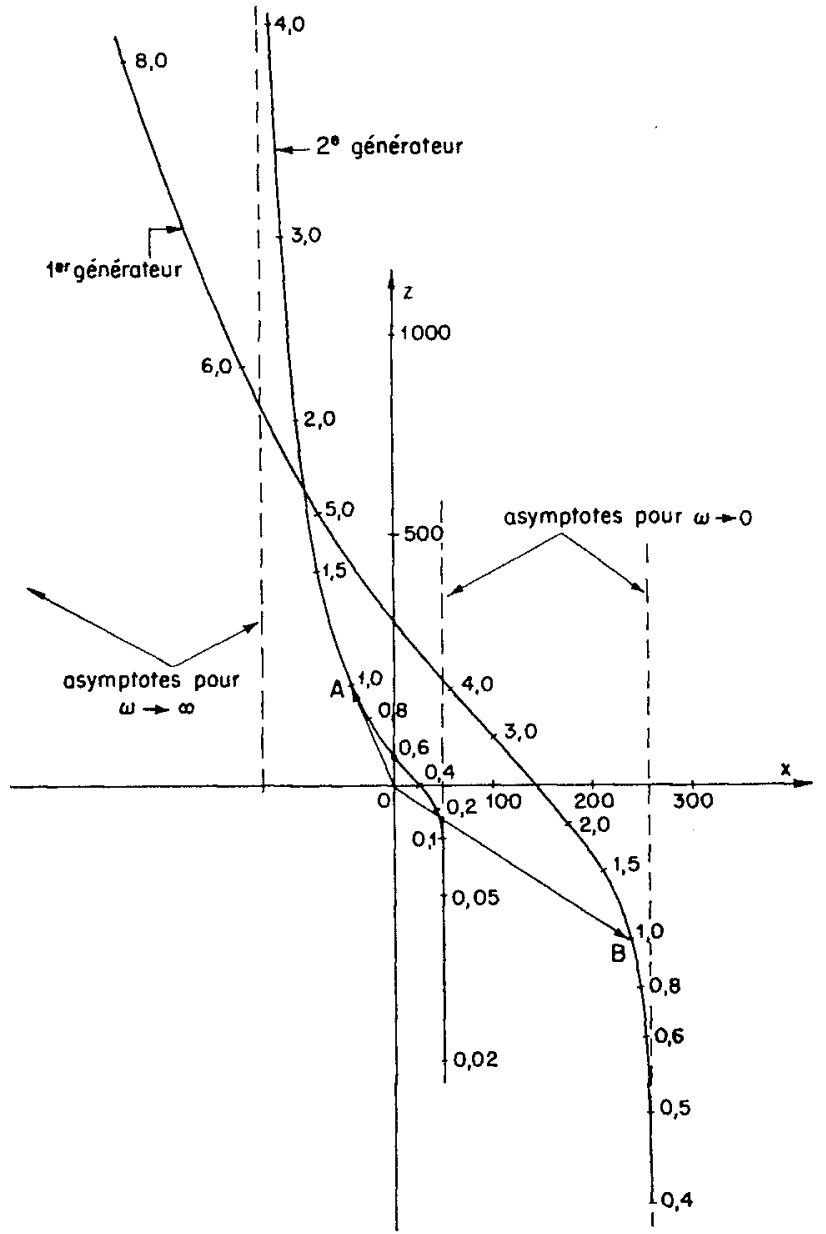

Fia. 7 


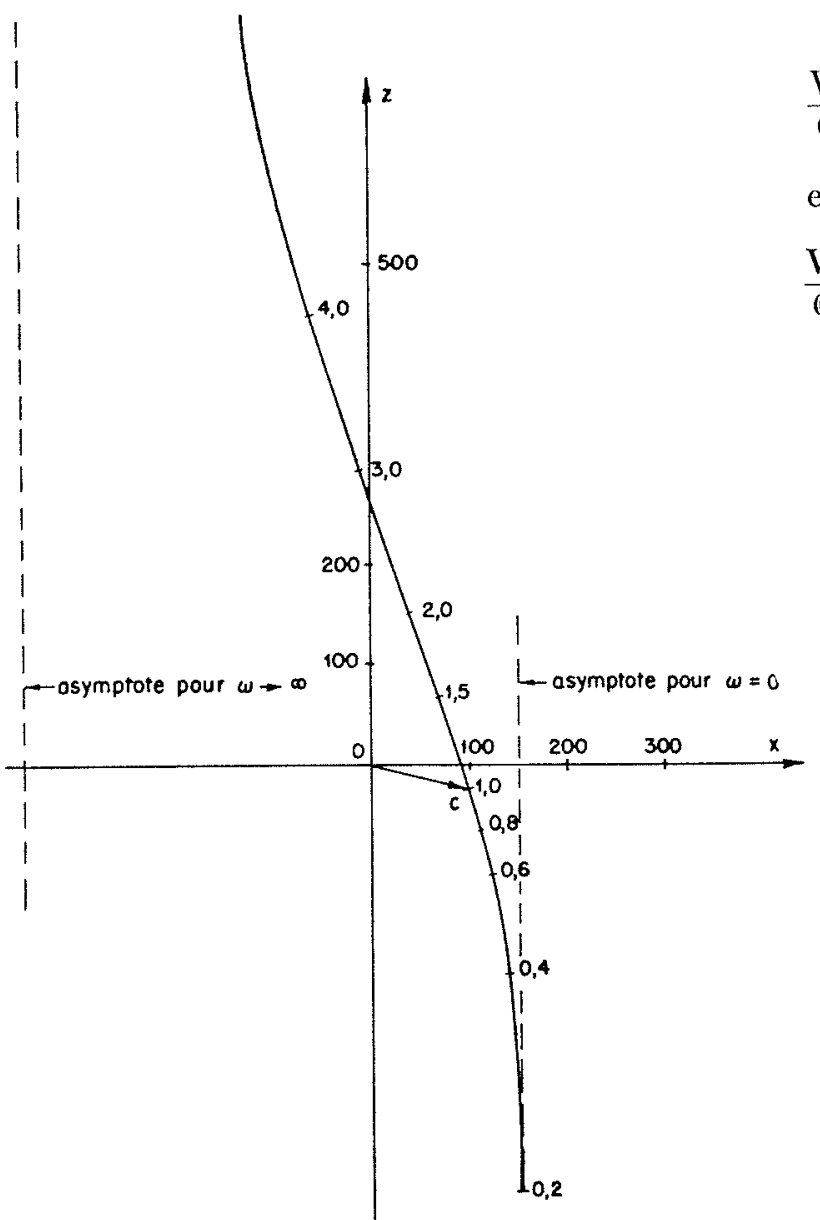

Fra. 8

Pour $\omega \rightarrow 0$ on obtient :

$Z(0) \rightarrow--\frac{\tau W_{n}}{\Theta Y_{0}} \frac{\nu}{\Theta Y_{0} \omega} ; \quad X(0) \rightarrow(u-3 / 2 v) \frac{\tau W_{n}}{\Theta Y_{0}}$

et pour $\omega \rightarrow \infty$ :

$$
Z(\infty) \rightarrow \frac{\tau W_{n}}{\Theta Y_{0}} \Theta Y_{0} \omega ; X(\infty) \rightarrow-2 u \cdot \frac{\tau W_{n}}{\Theta Y_{0}}
$$

Dans les installations normales on a :

d'ot̀ :

$$
\sigma>0, \mathrm{~T}_{r}>0 \text {, }
$$

$$
Z(0) \rightarrow-\infty ; Z(\infty) \rightarrow+\infty ; X(\infty)<0 .
$$

En couplant plusieurs installations de ce type, les mêmes conditions sont donc remplies et la condition de stabilité exige que le rayon vecteur balaye un angle de $+\pi$, ou que la courbe passe à droite de l'origine.

Comme au paragraphe II, le réseau constitué par le couplage des deux groupes est à la limite de la stabilité si la courbe de réponse passe par l'origine. Ceci a lieu si les grandeurs :

$$
\frac{W_{1} \tau_{1}}{\Theta_{1} Y_{1}} ; \frac{W_{2} \tau_{2}}{\Theta_{2} Y_{2}} ; \Theta_{1} Y_{1} ; \Theta_{2} Y_{2} ; u \text { et } v
$$

satisfont à une certaine relation que nous allons déterminer.
La courbe de réponse passe par l'origine si : $\frac{W_{1} \tau_{1}}{\Theta_{1} Y_{1}} x\left(\Theta_{1} Y_{1} \omega_{0} ; \mu ; \nu\right)+\frac{W_{2} \tau_{2}}{\Theta_{2} Y_{2}} x\left(\Theta_{2} Y_{2} \omega_{0} ; \mu ; \nu\right)=0$ et :

$\frac{W_{1} \tau_{1}}{\Theta_{1} Y_{1}} z\left(\Theta_{1} Y_{1} \omega_{0} ; \mu ; \nu\right)+\frac{W_{2} \tau_{2}}{\Theta_{2} Y_{2}} z\left(\Theta_{2} Y_{2} \omega_{0} ; u ; \nu\right)=0$

où $\omega_{0}$ est la pulsation de l'oscillation propre du réseau, qui est juste entretenue, ni amortie, ni amplifiée (cf. § II). Pour obtenir la relation correspondant à la limite de stabilité, on pourrait par exemple tirer $\omega_{0}$ de la relation (1) et porter sa valeur dans $(2)$. Ce calcul serait très compliqué. Nous allons procéder d'une façon plus simple.

Remarquons d'abord que si on pose $\Theta Y_{0} \omega=\alpha$ dans les expressions donnant $\mathrm{X} ; Z ; x$ et $z$, on s'aperçoit que tous les générateurs ayant même $\mu$ et $\nu$ ont même courbe $x=x(\alpha ; \mu ; \nu)$; $z=z(\alpha ; \mu ; \nu)$. Cette courbe est graduée en $\alpha$. Les graduations $\omega$ des deux courbes s'obtiennent respectivement par $\alpha /\left(\Theta_{1} Y_{1}\right)$ et $\alpha /\left(\Theta_{2} Y_{2}\right)$; si :

$$
\Theta_{1} Y_{1} \neq \Theta_{2} Y_{2}
$$

ces graduations $\omega$ sont donc différentes l'une de l'autre pour un même point.

Si le réseau est à la limite de la stabilité, il existe une pulsation $\omega_{0}$ telle que $\overrightarrow{\mathrm{OA}}+\overrightarrow{\mathrm{OB}}=0$ (fig. 9). Cette condition vectorielle peut être remplacée par deux conditions scalaires. Nous écrirons que $\overrightarrow{\mathrm{OA}}$ et $\overrightarrow{\mathrm{OB}}$ sont parallèles et opposés et que leurs projections sur OX sont égales en valeur absolue. La première condition donne :

ou :

$$
\frac{x\left(\Theta_{1} Y_{1} \omega_{0}\right)}{z\left(\Theta_{1} Y_{1} \omega_{0}\right)}=-\frac{x\left(\Theta_{2} Y_{2} \omega_{0}\right)}{z\left(\Theta_{2} Y_{2} \omega_{0}\right)}
$$

$$
\frac{x\left(\alpha_{1}\right)}{z\left(\alpha_{1}\right)}=-\frac{x\left(\alpha_{2}\right)}{z\left(\alpha_{2}\right)}
$$

avec $\alpha_{1}=\Theta_{1} Y_{1} \omega_{0}$ el $\alpha_{2.2}=\Theta_{2} Y_{2} \omega_{1)}$.

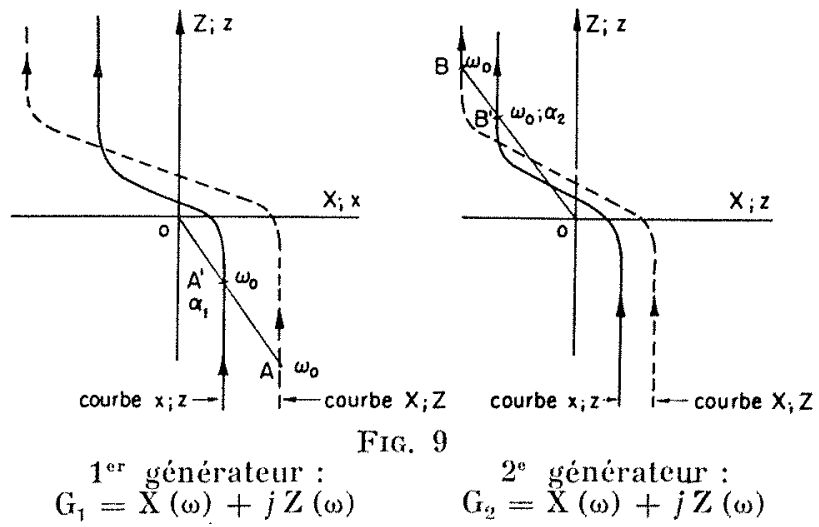




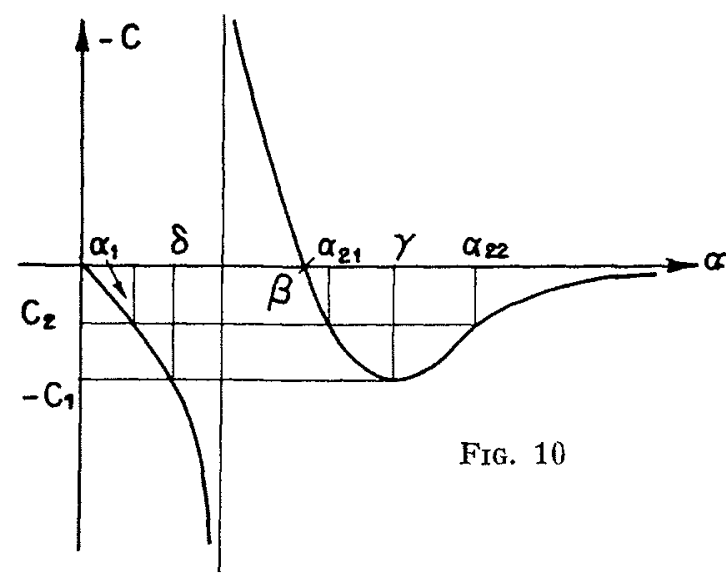

Il suffit de tracer $c(\alpha)=\frac{x(\alpha)}{z(\alpha)}$

où $c$ est la pente de la droite joignant le point $x ; z$ à l'origine.

Suivant sa pente, une droite passant par l'origine coupe la courbe $x$; $z$ en 1 ou 3 points. La figure 11 montre les différents cas.

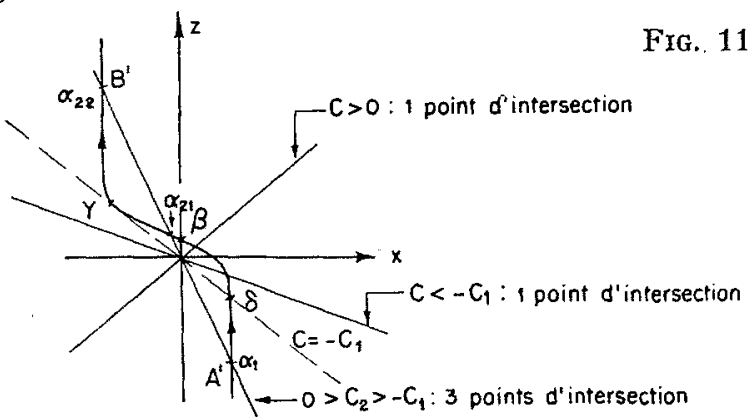

On obtient donc $\Theta_{2} Y_{2} \omega_{10}$ en fonction de $\Theta_{1} Y_{1} \omega_{0}$ en associant selon l'équation (3) les valeurs $\alpha_{1}$ et $\alpha_{22}$. On peut aussi déterminer $\Phi_{1} Y_{1} \omega_{0}=\alpha_{1}$ et $\Phi_{2} Y_{2} \omega_{0}=\alpha_{2}$ en fonction de

$$
\frac{\alpha_{1}}{\alpha_{2}}=\frac{\Theta_{1} Y_{1}}{\Theta_{2} Y_{2}}
$$

La condition $\left|\mathrm{X}_{1}\right|=\left|\mathrm{X}_{2}\right|$ peut s'écrire :

$\frac{W_{1} \tau_{1}}{\Theta_{1} Y_{1}}\left|\boldsymbol{x}\left(\Theta_{1} Y_{1} \omega_{0}\right)\right|=\frac{W_{2} \tau_{2}}{\Theta_{2} Y_{2}}\left|x\left(\Theta_{2} Y_{2} \omega_{0}\right)\right|$

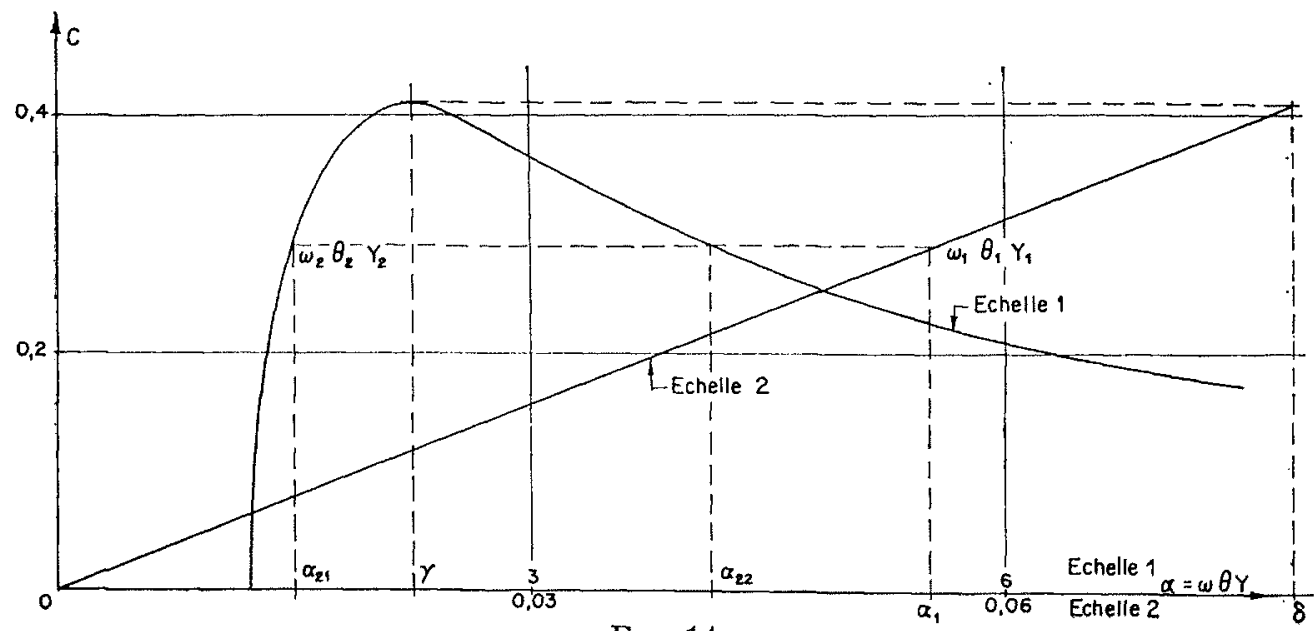

FIg. 14

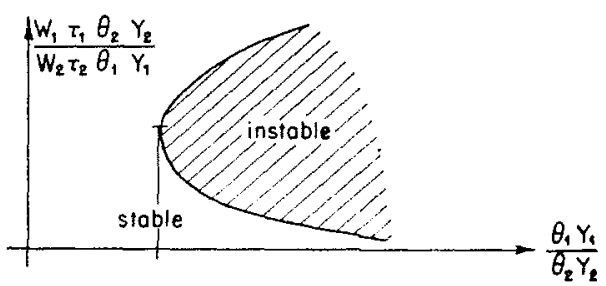

FIG. 12

D'où :

$$
\frac{W_{1} \tau_{1} \Theta_{2} Y_{2}}{W_{2} \tau_{2} \Theta_{1} Y_{1}}=\left|\frac{x\left(\Theta_{2} Y_{2} \omega_{0}\right)}{x\left(\Theta_{1} Y_{1} \omega_{0}\right)}\right|=\left|\frac{x\left(\alpha_{2}\right)}{x\left(\alpha_{1}\right)}\right|
$$

En remplaçant $\alpha_{1}$ et $\alpha_{2}$ par leur valeur en fonction de :

$$
\frac{\alpha_{1}}{\alpha_{2}}=\frac{\Theta_{1} Y_{1}}{\Theta_{2} Y_{2}}
$$

on peut mettre la relation sous la forme :

$$
\frac{W_{1} \tau_{1} \Theta_{2} Y_{2}}{W_{2} \tau_{2} \Theta_{1} Y_{1}}=f\left(\frac{\Theta_{1} Y_{1}}{\Theta_{2} Y_{2}}\right): \text { fig. } 12
$$

Pour déterminer quel est le domaine de stabilité, il suffit de remarquer que si $\Theta_{1} Y_{1}=\Theta_{2} Y_{2}$, on a toujours $\overrightarrow{O A}=\overrightarrow{O B}$; en effet, dans ce cas,

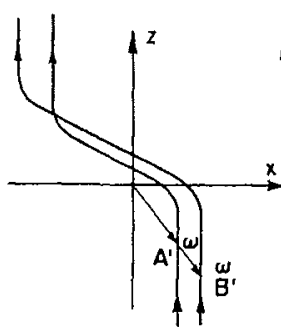

(a)

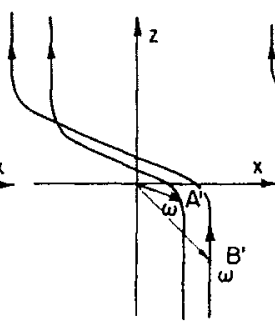

(b)

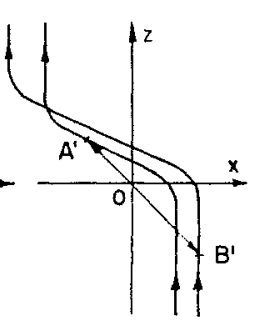

(c)
FIG. 13

(a) pen différents: stabilité $\Theta_{1} Y_{1} \stackrel{(a)}{=} \Theta_{2} Y_{2} \Theta_{1} Y_{1}$ et $\Theta_{2} Y_{2}$

(c) $\Theta_{1} Y_{1}$ et $\Theta_{2} Y_{0}$ inffisamment différents : it peut $\mathrm{y}$ avoir instabilite 
les courbes $x ; z$ portent les mêmes graduations; il y a donc toujours stabilité (fig. 13). On peut aller plus loin et dire qu'il ne peut y avoir instabilité que si $\Theta_{1} Y_{1}$ et $\Theta_{2} Y_{2}$ sont suffisamment différents l'un de l'autre (fig. 13).

Les figures 14 et 15 correspondent aux figures 10 et 12 avec $\mu=0,7$ et $\nu=0,1$.

On voit que, dans ce cas, il y a certainement stabilité si $1 / 25<\left(\Theta_{1} \mathrm{Y}_{1} /\left(\Theta_{2} \mathrm{Y}_{2}\right)<25 ;\left(\Theta_{1} \mathrm{Y}_{1}\right.\right.$ et $\Theta_{2} \mathrm{Y}_{2}$ jouent évidemment des rôles symétriques; on a supposé jusqu'ici que $\alpha_{1}=\Theta_{1} Y_{1}<\Theta_{2} Y_{2}=\alpha_{2}$, on obtient des résultats symétriques en posant $\alpha_{1}=\Theta_{2} Y_{2}$ et $\alpha_{2}=\Theta_{i} Y_{1}$.

En pratique, $\Theta$ varie de $0,3 \mathrm{~s}$ à $3 \mathrm{~s}$ environ, il ne peut donc pas $\mathrm{y}$ avoir instabilité si $\mathrm{Y}_{1}$ ou $\mathrm{Y}_{2}$ sont supérieurs à $40 \%$. Nous reviendrons ultérieurement sur ce cas et montrerons qu'il y a pratiquement toujours stabilité pourvu que les grandeurs $u$ et $v$ satisfassent à certaines inégalités.

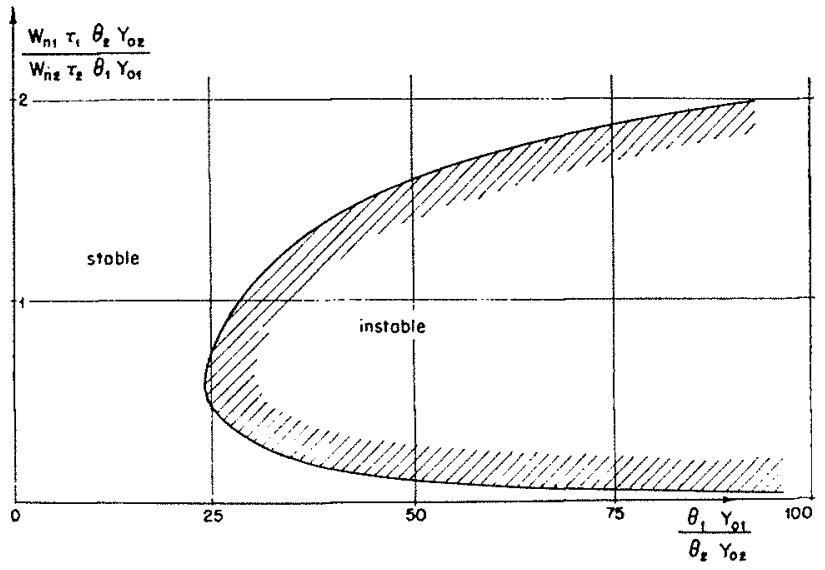

FIG. 15

c) Couplage de nombreuX GÉNÉrateurs DU TyPE FIGURE 1.

Nous venons de voir que le couplage de deux générateurs ne pouvait être instable que si leurs valeurs $\Theta Y$ étaient suffisamment différentes l'une de l'autre. En effet, les courbes de réponse ont toujours à peu près la même forme, mais la graduation en $\omega$ 《 glisse » le long de la courbe si $\Theta \mathrm{Y}$ varie et ce « glissement $\gg$ augmente avec $\Theta Y$ (cf. fig. 13).

Si on couple trois groupes, deux ayant des valeurs $\Theta \mathrm{Y}$ assez différentes et le troisième ayant une valeur $\Theta \mathrm{Y}$ moyenne par rapport aux deux premiers, on se rend facilement compte que ce troisième ne peut que stabiliser le couplage. En effet (fig. 16), on a vu que les deux premiers groupes ne pouvaient pas, en pratique, avoir des valeurs $\Theta Y$ telles que les points correspondants $A$ et $B$ sur les courbes de réponse soient très

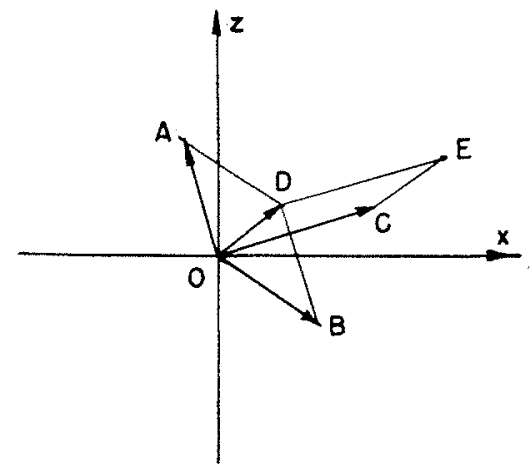

FIG. 16

éloignés. Le troisième groupe fournit done obligatoirement un vecteur OC' éloignant de l'origine la courbe de réponse du réseau total.

D'une façon générale, pour peu que les valeurs $\Theta Y$ ne soient pas concentrées au voisinage des valeurs extrêmes, qui sont de l'ordre de $0,3 \mathrm{~s}$ et $3 \mathrm{~s}$ environ, les réseaux contenant de nombreux groupes du type figure 1 sont donc toujours stables et même bien stables en général.

Nous allons montrer que de tels réseaux se comportent, du point de vue régulation, approximativement comme un groupe unique fictif. Pour cela, étudions d'abord l'expression :

$$
K=A f(a x)+B f(b x)+C f(c x)+\ldots
$$

On peut développer $f(a x)$ en série de Taylor comme suit :

$$
\begin{aligned}
& f(a x)=f[k x+(a-k) x]=f(k x) \\
& +(a-k) x f^{\prime}(k x)+1 / 2(a-k)^{2} x^{2} f^{\prime \prime}(k x)+\ldots
\end{aligned}
$$

En transformant de même $f(b x)$; $f(c x)$; etc., on obtient :

$$
\begin{aligned}
& \mathrm{K}=(\mathrm{A}+\mathrm{B}+\mathrm{C}+\ldots) f(k x) \\
& \quad+[\mathrm{A}(a-k)+\mathrm{B}(b-k)+\ldots] x f^{\prime}(k x) \\
& +1 / 2\left[\mathrm{~A}(a-k)^{2}+\mathrm{B}(b-k)^{2}+\ldots\right] x^{2} f^{\prime \prime}(k x)+\ldots
\end{aligned}
$$

Si on choisit $k$ tel que :

$$
\mathrm{A}(a-k)+\mathrm{B}(b-k)+\ldots=0
$$

et si les lermes relatifs aux dérivées d'ordre supérieur sont suffisamment petits, on peut écrire :

$$
\mathrm{K} \#(\mathrm{~A}+\mathrm{B}+\mathrm{C}+\ldots) f(k x)
$$

et $k$ est donné par :

$$
\frac{\mathrm{A} a+\mathrm{B} b+\mathrm{C} c+\ldots}{\mathrm{A}+\mathrm{B}+\mathrm{C}+\ldots}
$$


L'erreur relative due au terme en $f^{\prime \prime}$ est alors :

$$
\begin{array}{r}
\eta=\frac{\mathrm{A} a^{2}+\mathrm{B} b^{2}+\ldots-2(\mathrm{~A} a+\mathrm{B} b+\ldots) k+(\mathrm{A}+\mathrm{B}+\mathrm{C}+\ldots) k^{2}}{\mathrm{~A}+\mathrm{B}+\mathrm{C}+\ldots} 1 / 2 x^{2} \frac{f^{\prime \prime}(k x)}{f(k x)} \\
\quad=\frac{\mathrm{A} a^{2}+\mathrm{B} b^{2}+\mathrm{C} c^{2}+\ldots-(\mathrm{A}+\mathrm{B}+\mathrm{C} \ldots) k^{2}}{\mathrm{~A}+\mathrm{B}+\mathrm{C}+\ldots} 1 / 2 x^{2} \frac{f^{\prime \prime}(k x)}{f(k x)}
\end{array}
$$

Dans les applications que nous aurons à faire de ces formules, $f(k x)$ est, au voisinage de chaque valeur $x$, approximativement représentable par $\alpha(k x)^{n}$ avec $-1<n<+2$. D'où dans ce cas: $k^{2} x^{2} f^{\prime \prime}(k x)<2 f(k x)$.

On en déduit :

$$
\eta<\frac{\mathrm{A} a^{2}+\mathrm{B} b^{2}+\ldots}{k^{2}(\mathrm{~A}+\mathrm{B}+\ldots)}-1
$$

Si cette erreur est petite, on peut admettre dans notre cas que les autres erreurs dues aux termes $f^{(n)}(k x)$ d'ordre plus élevé sont encore bien plus petites.

Appliquons maintenant ces formules au couplage de plusieurs groupes. Dans ce cas, on avait :

$\mathrm{X}(\omega)=\mathrm{X}_{1}(\omega)+\mathrm{X}_{2}(\omega)+\ldots$ et de nuême pour $Z(\omega)$

Dans le cas de groupes du type figure 1, on peut écrire :

$$
\begin{aligned}
& \mathrm{X}=\frac{\mathrm{W}_{1} \tau_{1}}{\Theta_{1} \mathrm{Y}_{1}} x\left(\Theta_{1} \mathrm{Y}_{1} \omega ; \mu_{1} ; \nu_{1}\right) \\
&+\frac{W_{2} \tau_{2}}{\Theta_{2} Y_{2}} x\left(\Theta_{2} Y_{2} \omega ; \mu_{2} ; \nu_{2}\right)+\ldots
\end{aligned}
$$

et de même pour $Z$.

D'où, en appliquant les relations ci-dessus :

$$
\begin{aligned}
\mathrm{X \# (}\left(\frac{\mathrm{W}_{1} \tau_{1}}{\Theta_{1} \mathrm{Y}_{1}}+\frac{\mathrm{W}_{2} \tau_{2}}{\Theta_{2} \mathrm{Y}_{2}}\right. & +\ldots) x(\Theta \mathrm{Y} \omega ; \mu ; \nu) \\
& =\frac{\Theta \mathrm{Y}}{\mathrm{W} \tau} x(\Theta \mathrm{Y} \omega ; \mu ; \nu)
\end{aligned}
$$

avec :

$$
\begin{aligned}
\Theta Y & =\frac{W_{1} \tau_{1}+W_{2} \tau_{2}+\ldots}{\left(W_{1} \tau_{1}\right) /\left(\Theta_{1} Y_{1}\right)+\left(W_{2} \tau_{2}\right) /\left(\Theta_{2} Y_{2}\right)+\ldots} \\
\text { et } W_{\tau} & =W_{1} \tau_{1}+W_{2} \tau_{2}+\ldots
\end{aligned}
$$

Les erreurs relatives des abscisses et ordonnées des points de la courbe de réponse se calculent aisément. L'erreur due aux valeurs $\Theta Y$ est, par exemple :
Pour se rendre compte de cette erreur, nous supposerons que les groupes travaillent à peu près tous à pleine charge (ce qui est le cas normal), et que $\tau_{1} / \Theta_{1} \# \tau_{2} / \Theta_{2} \# \ldots$ (ce qui est vrai en ordre de grandeur). D'où :

$$
\eta \# \frac{\left(W_{1} \Theta_{1}^{2}+W_{2} \Theta_{2}^{2}+\ldots\right)\left(W_{1}+W_{2}+\ldots\right)}{\left(W_{1} \Theta_{1}+W_{2} \Theta_{2}+\ldots\right)^{2}}-1
$$

$\eta$ est d'autant plus grand que les valeurs de $\Theta$ sont plus différentes. Pour $\Theta_{1}=\Theta_{2}=\ldots$ on obtient évidemment $\eta=0$. Ceci peut aussi être déduit directement de la relation (4). Si $a \# b \# c \#$... ces valeurs sont évidemment trés voisines de $k$ et $a-k \# 0 ; b-k \# 0 \ldots$

Pour évaluer $\eta$ dans un cas particulier, nous étudierons un réseau dans lequel la somme des puissances des générateurs ayant une valeur $\Theta$ comprise entre $\Theta_{1}$ et $\Theta_{1}+\Delta \Theta$ ne dépend pas de $\Theta_{1}$ pourvu que $\Theta_{2}<\Theta_{1}<\Theta_{3}$. Pratiquement, cela veut dire que la somme des puissances des centrales telles que $0,5 \mathrm{~s}<\Theta<0,7 \mathrm{~s}$ est la même que celle des centrales telles que $0,7 \mathrm{~s}<\Theta<0,9 \mathrm{~s}$ ou $0,9 \mathrm{~s}<\Theta<1,1 \mathrm{~s}$, etc. Dans ce cas particulier, on obtient :

$$
\eta<\frac{\int_{\Theta_{3}}^{\theta_{s} \Theta^{2}} d \Theta \times \int_{\theta_{2}}^{\theta_{3}} d \Theta}{\left(\int_{\theta_{2}}^{\left.\theta_{3} \Theta d \Theta\right)^{2}}\right.}-1
$$

$$
=(4 / 3) \frac{1+\left(\Theta_{3} / \Theta_{2}\right)+\left(\Theta_{3} / \Theta_{2}\right)^{2}}{1+2\left(\Theta_{3} / \Theta_{2}\right)+\left(\Theta_{3} / \Theta_{2}\right)^{2}}-1
$$

En général, la majeure partie de la puissance d'un réseau est telle que $0,5 \mathrm{~s}<\Theta<1,5 \mathrm{~s}$ ou $0,4 \mathrm{~s}<\Theta<1,6 \mathrm{~s}$. On obtient dans ces deux cas respectivement $\eta<8 \%$ et $\eta<12 \%$. Dire que les valeurs de $\Theta$ sont relativement voisines revient à dire que le réseau est très stable, d'après ce que nous avons vu. Inversement on peut dire qu'un réseau peut être remplacé par un seul groupe fictif si ce réseau est suffisamment stable. D'ailleurs, dans le cas contraire, il est évident que cette approximation est fausse puisqu'elle tendrait à démontrer que le réseau est stable alors qu'il ne l'est pas par hypothèse. (Dans ce cas, $\eta$ serait très grand.)

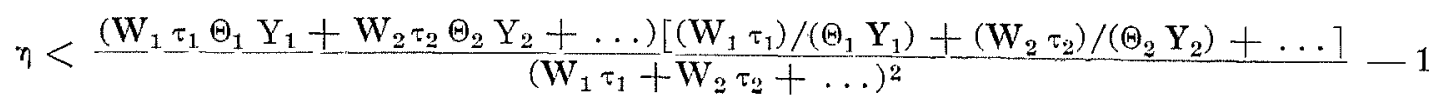


d) QUalités DU RÉGLAGE DANS UN RÉSEAU INTERCONNECTÉ DU TYPE ÉTUDIÉ JUSQU'ICI.

Nous montrerons, dans l'annexe I, que le carré de l'erreur moyenne de réglage de la fréquence dans un réseau ne contenant qu'un seul groupe est de la forme :

$$
\varepsilon^{2}=\frac{\Theta^{3}}{(W \tau)^{2}} \cdot(\Delta W)^{2} f(\mu ; \nu),
$$

où $\Delta W$ est l'amplitude moyenne des variations de charge électrique et $W$ la puissance du groupe. Soient $u=\mu_{0} ; \nu=v_{0}$, les valeurs de $u$. et $v$ donnant la plus petite erreur moyenne $\varepsilon$.

Nous allons ici simplement comparer deux cas de dimensionnement des valeurs de $\mu$. et $\nu$ des régulateurs dans un réseau interconnecté.

On peut d'abord choisir $u=\mu_{0}$ et $\nu=\nu_{0}$ pour chaque groupe. Ceci constituerait le dimensionnement optimum pour obtenir l'erreur $\varepsilon$ la plus petite si la théorie du réglage en réseau séparé s'appliquait aux réseaux interconnectés. Dans ce cas, l'erreur de réglage serait donnée par :

$$
\varepsilon_{1}^{2}=\frac{\Theta^{2}}{(W \tau)^{2}}(\Delta W)^{2} f\left(\mu_{0} ; \nu_{0}\right)
$$

où $\Theta$ et $W \tau$ sont les caractéristiques du groupe fictif, définies plus haut.

\section{Donc :}

$\varepsilon_{1}^{2}=\frac{W_{1} \tau_{1}+W_{2} \tau_{2}+\ldots}{\left[\left(W_{1} \tau_{1} / \Theta_{1}\right)+\left(W_{2} \tau_{2} / \Theta_{2}\right)+\ldots\right]^{3}}(\Delta W)^{2} f\left(\mu_{0} ; v_{0}\right)$

On peut ensuite faire marcher tous les groupes sur «limiteur d'ouverture», sauf celui ayant la valeur $\Theta$ la plus petite. Ceci revient à faire $\mu=\nu=0$ pour tous les autres groupes : les régleurs n'interviennent plus. Dans ce cas, on obtient facilement et directement la fonction de réponse du réesau :

$$
\begin{aligned}
G(j \omega)= & \left(W_{1} \tau_{1}+W_{2} \tau_{2}+\ldots\right) j \omega \\
& +\frac{W_{1} \tau_{1}}{\Theta_{1}} \frac{1-\Theta_{1} j \omega}{1+(1 / 2) \Theta_{1} j \omega} \frac{\nu_{1}+\mu_{1} \Theta_{1} j \omega}{\Theta_{1} j \omega}
\end{aligned}
$$

où l'indice 1 se rapporte au groupe non sur limiteur. On peut aussi écrire :

$$
\begin{aligned}
G(j \omega)= & \frac{W_{1} \tau_{1}+W_{2} \tau_{2}+\ldots}{\Theta_{1}} \\
& {\left[\Theta_{1} j \omega+\frac{1-\Theta_{1} j \omega}{1+1 / 2 \Theta_{1} j \omega} \cdot \frac{v^{\prime}+\omega^{\prime} \Theta_{1} j \omega}{\Theta_{1} j \omega}\right] }
\end{aligned}
$$

avec :

$$
v^{\prime}=v_{1} \frac{W_{1} \tau_{1}}{W_{1} \tau_{1}+W_{2} \tau_{2}+\ldots}
$$

et de même pour $\mu^{\prime}$.

Le réseau est donc, dans ce cas, en toute ri- gueur équivalent à un groupe unique dont $\Theta=\Theta_{1}$ et $W_{\tau}=W_{1} \tau_{1}+W_{2} \tau_{2}+\ldots$ Si on choisit $\nu_{1}$ et $u_{1}$ tels que $\nu^{\prime}=\nu_{0}$ et $\mu^{\prime}=y_{0}$, l'erreur de réglage est donnée par :

$$
\varepsilon_{2}^{2}=\frac{\Theta_{1}{ }^{3}}{\left(W_{1} \tau_{1}+W_{2} \tau_{2}+\ldots\right)^{2}} \cdot(\Delta W)^{2} f\left(u_{0} ; v_{0}\right)
$$

On peut facilement montrer que $\varepsilon_{2}<\varepsilon_{1}$. En effet, $\Theta_{1}$ étant la plus petite parmi les valeurs de $\Theta$, on a :

$$
\begin{aligned}
\left(\frac{W_{1} \tau_{1}}{\Theta_{1}}+\frac{W_{2} \tau_{2}}{\Theta_{1}}\right. & \left.+\frac{W_{3} \tau_{3}}{\Theta_{1}}+\ldots\right)^{3} \\
& >\left(\frac{W_{1} \tau_{1}}{\Theta_{1}}+\frac{W_{2} \tau_{2}}{\Theta_{2}}+\ldots\right)^{3}
\end{aligned}
$$

D'où :

$$
\begin{aligned}
& \frac{W_{1} \tau_{1}+W_{2} \tau_{2}+\ldots}{\left[\left(W_{1} \tau_{1} / \Theta_{1}\right)+\left(W_{2} \tau_{2} / \Theta_{2}\right)+\ldots\right]^{3}} \\
& \quad>\frac{\Theta_{1}^{3}}{\left(W_{1} \tau_{1}+W_{2} \tau_{2}+\ldots\right)^{2}}
\end{aligned}
$$

c'est-à-dire : $\varepsilon_{1}^{2}>\varepsilon_{2}^{2}$.

Ceci prouve que la théorie du réglage en réseau séparé ne peut pas servir à dimensionner les régulateurs dans un réseau interconnecté.

Evidemment, la solution consistant à mettre sur limiteur tous les groupes, sauf ceux ayant des valeurs $\Theta$ petites, n'est pas elle-même la solution optimum.

Néanmoins on voit que plus un groupe possède un temps $\Theta$ élevé, plus on a intérêt à choisir les paramètres $\mu$ et $v$ de son régleur petits et inversement. En particulier, dans la solution décrite plus haut, $\varphi_{0}$ et $\nu_{1}$ sont donnés par :

$$
\mu_{1}=\mu_{0} \frac{W_{1} \tau_{1}+W_{2} \tau_{2}+\ldots}{W_{1} \tau_{1}}
$$

et de même pour $v_{1}$. Nous montrerons par la suite qu'on peut effectivement mettre les groupes ayant un temps $\Theta$ très grand sur limiteur. Par contre, nous montrerons aussi qu'il faut munir les groupes ayant un temps $\Theta$ petit de régleurs dont les paramètres $y$ et $v$ sont grands, c'est-à-dire les paramètres $\sigma=(\Theta / \tau \mu)$ et $\mathrm{T} r=\Theta(\mu / \nu)$ petits. Ceci nécessite que ces régleurs soient de très bonne qualité et que les imperfections, jeux, frottements, temps de retard, périodes propres, etc., des diverses parties des régleurs soient petits devant $\sigma, \mathrm{T}_{r}$ et les quantités qui en découlent.

e) Méthode de LA Covrbe symétrique pouk L'ÉTUDE DU COUPLAGE DF DEUX RÉSEAUX PARTIELS (OU DE DEUX GÉNÉRATEURS) *

Nous appellerons - dans ce paragraphe seulement - courbe normale la courbe de réponse

( ) Cette méthode a une certaine parenté avec une des methodes dites « de Nasse $\$$. 
et courbe symétrique la courbe symétrique par rapport à l'origine de la courbe de réponse.

Etudions le couplage de deux réseaux partiels en traçant la courbe normale de l'un et la courbe symélrique de l'autre (fig. 17):

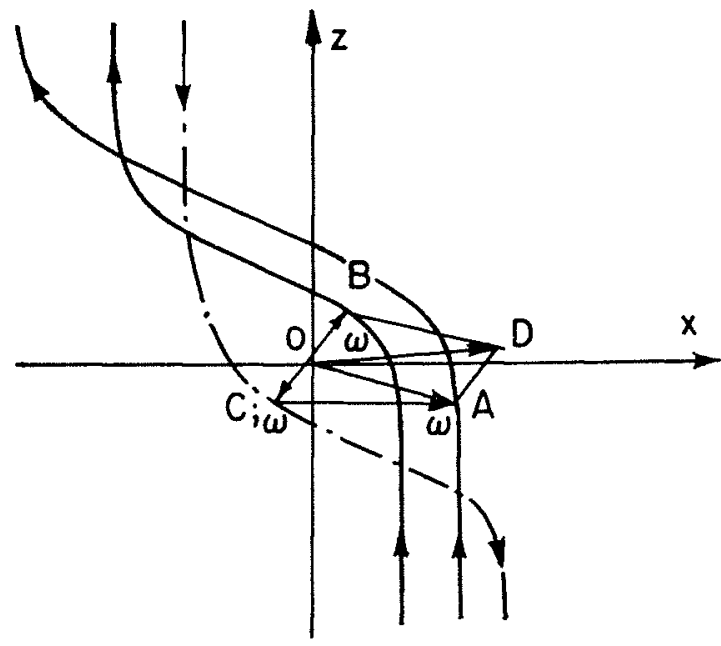

FIG. 17

Soient A, B et C les divers points de graduation $\omega$, l'égalité des triangles ODA et ACO montre que $\overrightarrow{\mathrm{OD}}$ et $\overrightarrow{\mathrm{CA}}$ sont parallèles. Au lieu de déterminer l'angle de rotation du vecteur $\overrightarrow{\mathrm{OD}}$ conme il a été dit aux paragraphes II et III, on peut aussi étudier la rotation du vecteur $\overrightarrow{\mathrm{CA}}$. Cette rotation ne dépend que des graduations $\omega$ des points d'intersection de la courbe normale de l'un des réseaux avec la courbe symétrique

de l'autre. Comme sur la figure 17 , le vecteur $\overrightarrow{\mathrm{CA}}$ partira toujours du point de la courbe symétrique et l'indice « 1 se rapportera à cette courbe, alors que l'indice $\ll 2 »$ se rapportera à la courbe normale.

La figure 18 montre ce qui peut se passer à un point d'intersection.

Comme les signes des angles marqués sur chaque figure restent les mêmes si on fait tourner la figure, on voit que la figure 18 donne tous les cas possibles.

Si $\omega_{1}$ et $\omega_{2}$ sont les graduations des deux courbes à leur point d'intersection, le signe de l'angle $\left(a b ; a c\right.$ ) et le signe de $\omega_{1}-\omega_{2}$ (ou le sens de l'inégalité de $\left.\omega_{1} \gtrless \omega_{2}\right)$ permettent de connaître le signe de l'angle $\left(a b ; a^{\prime} d\right)$. En effet, on a :

$(a b ; a c)>0 ; \omega_{1}<\omega_{2}:\left(a b ; a^{\prime} d\right)>0(\mathrm{fig} .18 \mathrm{~A})$ $(a b ; a c)>0 ; \omega_{1}>\omega_{2}:\left(a b ; a^{\prime} d\right)<0$ (fig. 18 B) $(a b ; a c)<0 ; \omega_{1}>\omega_{2}:\left(a b ; a^{\prime} d\right)<0$ (fig. $18 \mathrm{C}$ ) $(a b ; a c)<0 ; \omega_{1}<\omega_{2}:\left(a b ; a^{\prime} d\right)>0$ (fig. 18 D)

D’une façon générale, la stabilité ou l'instabilité se conservent quand on déforme les courbes pourvu que, pendant la déformation, il ne se pro-
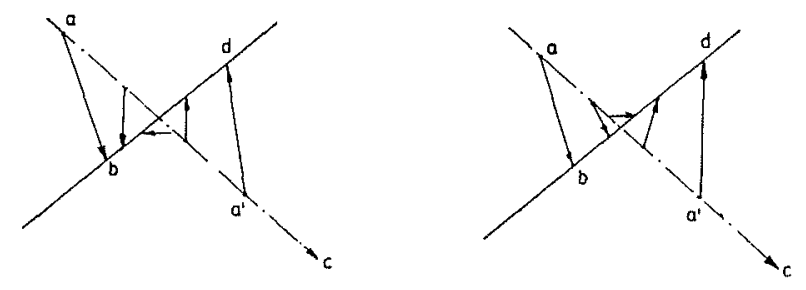

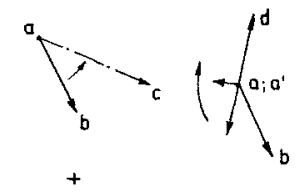

(A)
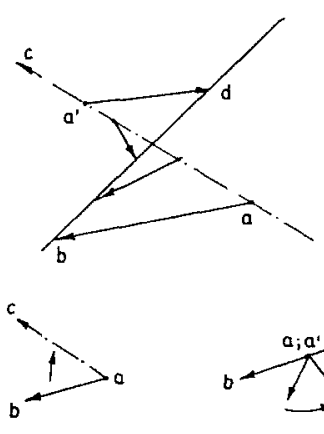

$-$

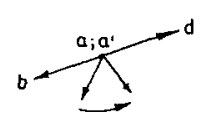

(c)

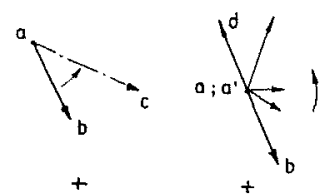

(B)
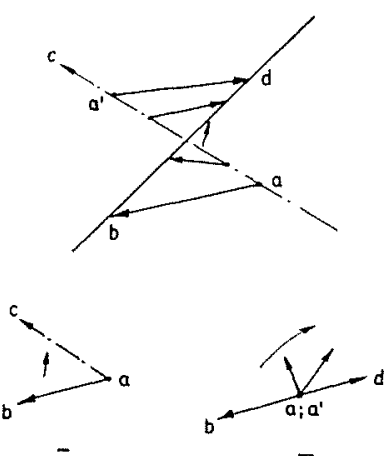

$-$
FIG. 18

duise pas de déchirure ou de recoupement nouveau entre les deux courbes et que les recoupements anciens se conservent avec leur signe de $\omega_{1}-\omega_{2}$. En effet, les angles de rotation du vecteur $\overrightarrow{\mathrm{CA}}$ (fig. 17) se conservent. On peut même éliminer les points doubles de chaque courbe par déformation continue pourvu que les recoupements soient respectés.

Pour obtenir l'angle de rotation total de $\overrightarrow{\mathrm{CA}}$, il faut parfois ne considérer que certains points d'intersection. Pour étudier celte question, nous numéroterons les points d'intersection de façon à ce cque $\omega_{11}<\omega_{12}<\omega_{13}<\omega_{14}<\ldots$ ces valeurs étant les graduations de la courbe symétrique aux points d'intersection. Si on range alors l'ensemble des graduations $\omega$ relatives aux points d'intersection par ordre croissant, seuls les points poux lesquels les deux valeurs de $\omega: \omega_{1 n}$ et $\omega_{2 n}$ sont voisines sont à considérer. ILa figure 19 montre des exemples.

Dans le cas de la figure $19 \mathrm{~A}$, on a : $\omega_{23}<\omega_{11}<\omega_{22}<\omega_{21}<\omega_{13}$. Seules les graduations $\omega_{1 n}$ et $\omega_{2 n}$ pour $n=2$ sont voisines. On voit, en effet, qu'en modifiant la figure, pour avoir par exemple $\omega_{11}<\omega_{23}<\omega_{22}<\omega_{12}<\omega_{21}<\omega_{13}$ l'angle balay'é par le rayon vecteus ne varie pas. Par contre, dans le cas de la figure $19 \mathrm{~B}$, on a : 


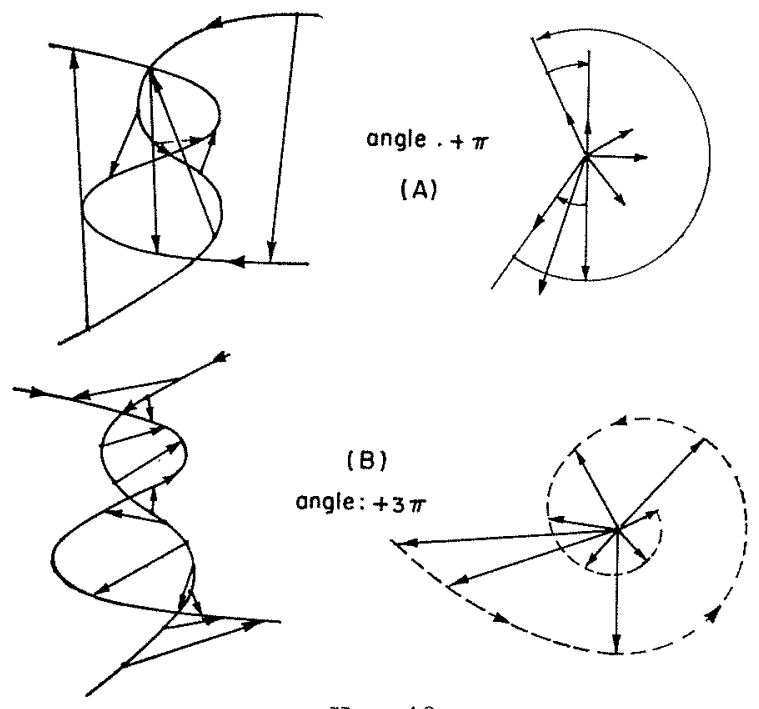

FIG. 19

$\omega_{21}<\omega_{11}<\omega_{12}<\omega_{22}<\omega_{23}<\omega_{13}$ et les graduations $\omega_{1 n}$ et $\omega_{2 n}$ sont voisines pour tous les points d'intersection $n=1 ; 2 ; 3$. L'angle total peut être : $+3 \pi ;-3 \pi ;+\pi$ ou $-\pi$ selon les signes obtenus à chaque point d'intersection. Chaque point d'intersection intervenant fournit un angle de $\pm \pi$ selon le signe obtenu précédemment. Il peut arriver qu'aucun point d'intersection ne soit à considérer; ceci est par exemple le cas si on a $\omega_{11}<\omega_{22}<\omega_{21}<\omega_{12}$. En dehors des angles dus aux points d'intersection, il faut considérer les angles dus à $\omega=0$ et $\omega=\infty$. Dans le cas des courbes de réponse de réseaux électriques, nous démontrerons que $\lim \mathrm{X}(\omega)$ et $\lim \mathrm{X}(\omega)$ sont finies, par contre $\lim _{\omega \rightarrow 0} Z(\omega)=-\infty$ et $\lim _{\omega \rightarrow \infty} Z(\omega)= \pm=\infty$. Les angles à ajouter pour $\omega=0$ et $\omega=\infty$ sont les suivants :

$$
\begin{aligned}
& Z_{1}(\infty)=+\infty\left\{\begin{array}{l}
-\mathrm{X}_{1}(0)<\mathrm{X}_{2}(\infty) ;-\mathrm{X}_{1}(\infty)<\mathrm{X}_{2}(0):+\pi \\
-\mathrm{X}_{1}(0)<\mathrm{X}_{2}(\infty) ;-\mathrm{X}_{1}(\infty)>\mathrm{X}_{2}(0) ; \quad 0 \\
-\mathrm{X}_{1}(0)>\mathrm{X}_{2}(\infty) ;-\mathrm{X}_{1}(\infty)<\mathrm{X}_{2}(0): 0 \\
-\mathrm{X}_{1}(0)>\mathrm{X}_{2}(\infty) ;-\mathrm{X}_{1}(\infty)>\mathrm{X}_{2}(0):-\pi
\end{array}\right. \\
& Z_{1}(\infty)=-\infty \\
& Z_{2}(\infty)=+\infty\left\{\begin{array}{l}
-\mathrm{X}_{1}(\infty)<-\mathrm{X}_{1}(0)<\mathrm{X}_{2}(\infty):+\pi \\
-\mathrm{X}_{1}(0)<-\mathrm{X}_{1}(\infty)<\mathrm{X}_{2}(\infty):+\pi \\
-\mathrm{X}_{1}(0)<\mathrm{X}_{2}(\infty)<-\mathrm{X}_{1}(\infty): 0 \\
-\mathrm{X}_{1}(\infty)<\mathrm{X}_{2}(\infty)<-\mathrm{X}_{1}(0): 0 \\
\mathrm{X}_{2}(\infty)<-\mathrm{X}_{1}(0)<-\mathrm{X}_{1}(\infty):-\pi \\
\mathrm{X}_{2}(\infty)<-\mathrm{X}_{1}(\infty)<-\mathrm{X}_{1}(0):-\pi
\end{array}\right. \\
& Z_{1}(\infty)=-\infty \\
& Z_{2}(\infty)=-\infty \\
& \text { toujours instable. }
\end{aligned}
$$

La condition de stabilité est la suivante : la somme des angles doit être égale à $+\pi$ et lim $Z(\omega)$ doit être $+\infty$ pour le réseau d'ensemble. Cette dernière condition $[Z(\infty)=+\infty]$ est en particulier vérifiée si les deux réseaux partiels sont stables avant couplage.

On voit que la limite de stabilité est atteinte si $\omega_{1 n}=\omega_{2 n}$ pour l'un des points d'intersection. Ceci est évident sur la figure $17:$ si $\overrightarrow{\mathrm{OA}}=\overrightarrow{\mathrm{OC}}$, on a $\overrightarrow{\mathrm{OA}}+\overrightarrow{\mathrm{OB}}=0$ et la courbe de réponse de l'ensemble passe par l'origine. En pratique, dans les réseaux habituels, il n'y a guère que zéro ou un point d'intersection à considérer. En effet, si on fait croitre $\omega$ à partir de zéro, la courbe normale vient de $Z=-\infty$ el la courbe symétrique de $Z=+\infty$. On a donc, en général, ur cas analogue à la figure $19 \mathrm{~A}$.

Cette méthode est surtout intéressante si l'un des réseaux partiels est donné, tandis que dans l'autre on peut faire varier un ou plusicurs paramètres.

\section{V. - CONCLUSIONS DE LA PREMIẼRE PARTIE}

Dans cette première partie, nous avons présenté une méthode d'étude du réglage de la fréquence des réseaux interconnectés. Cette méthode - telle qu'elle est décrite jusqu'ici - ne s'applique qu'aux réseaux satisfaisant aux quatre conditions suivantes :

a) Le réglage des lensions des alternateurs est parfait sous tous rapports et les impédances de liaison entre les groupes sont négligeables;

b) On connaît effectivement les équations régissant les phénomènes du réglage de la fréquence; c) Ces équations sont linéaires ou linéarisabies sans trop d'erreurs;

d) La fréquence est la même partout à chaque instant.

Dans les chapitres suivants, nous allons lever l'une après l'autre ces restrictions. Les phénomènes purement électriques seront pris en compte et nous montrerons dans quelles conditions ils interviennent d'une façon notable pour le réglage de la fréquence. Les non-linéarités seront également prises en compte et finalement nous étudierons de quelle façon on peut se ser- 
vir de données expérimentales pour trouver les équations de base dont on a besoin.

La méthode présentée dans cette première partie n'a servi jusqu'ici qu'à étudier la stabilité du réglage. Dans les chapitres suivants, nous étudierons le degré de stabilité du réglage et surtout la précision du réglage. En effet, la stabilité n'est qu'une qualité nécessaire, mais absolument insuffisante pour qu'un réglage soit correct; pour ceci, il faut que le réglage soit suffisamment précis. Inversement, si le réglage est suffisamment précis, il est nécessairement stable, au sens des oscillations des équations linéaires.

Dans cette première partie, nous avons présenté un certain nombre d'applications de la méthode exposée. Les résultats trouvés restent intéressants en dépit des restrictions que nous venons d'énoncer et des trois hypothèses spéciales que nous avons faites dans ces applications. En dehors des trois hypothèses générales énumérées plus haut, nous avons admis:

a) Que le statisme permanent avait une influence négligeable;

b) Que l'autoréglage dû aux collines des turbines était nul;

c) Que la hauteur de chute ne dépassait pas $100 \mathrm{~m}$ environ.

On montrera par la suite que les deux premières ne changent effectivement pas les conclusions suivantes valables pour les oscillations dites moyennes, c'est-à-dire dont la période est de l'ordre de grandeur de 1 à 20 secondes environ : a) Un réseau formé d'un ensemble de groupes habituels, isolément stables est lui-même stable;

b) Un réséau interconnecté stable est approximativement équivalent à un groupe unique fictif dont les caractéristiques sont définies dans le texte;

c) La théorie du réglage des réseaux ne contenant qu'un seul groupe, ne peut pas servir à dimensionner les paramètres des régulateurs de façon à obtenir la précision de réglage optimum;

d) La précision du réglage de la fréquence d'un réseau interconnecté peut être bien meilleure que celle d'un réseau séparé, même si les fluctuations relatives de puissance sont les mêmes;

e) Pour obtenir la précision de réglage optimum, on doit mettre les groupes ayant un temps $\Theta=(\Sigma \mathrm{L} \mathrm{V}) / g \mathrm{H}$ élevé sur limiteur d'ouverture et munir les groupes ayant un faible temps $\Theta$ de régulateurs ayant des qualités supérieures à celles requises pour le réglage des réseaux séparés.

On montrera aussi, par la suite, qu'on peut faire abstraction, pour l'étude, des oscillations moyennes des cheminées d'équilibre et des galeries à leur amont, des canaux, etc., dont les temps caractéristiques d'oscillations sont suffisamment grands, de même que des phénomènes dont les temps caractéristiques sont suffisamment courts.

(A suivre.)

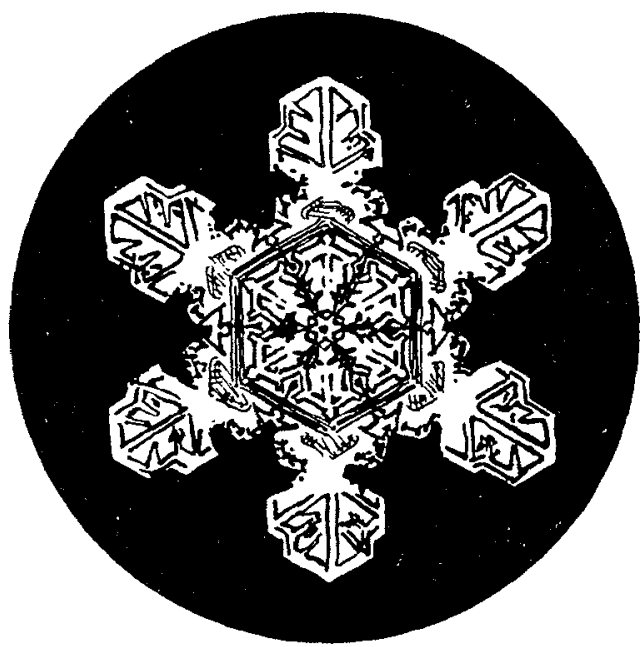

